Research Article Plant Genetics

\title{
Orf165 is associated with cytoplasmic male sterility in pepper
}

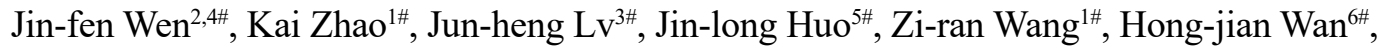 \\ Hai-shan Zhu ${ }^{1}$, Zhu-qing Zhang ${ }^{3}$, Gui-fang Shao ${ }^{1}$, Jiao Wang ${ }^{1}$, Shui Zhang ${ }^{1}$, Ting-yu Yang ${ }^{1}$, \\ Jing-rou Zhang ${ }^{1}$, Xue-xiao Zou ${ }^{3}$, Ming-hua Deng ${ }^{1,4}$ (D) \\ ${ }^{I}$ College of Horticulture, Yunnan Agricultural University, Kunming, China. \\ ${ }^{2}$ Faculty of Architecture and City Planning, Kunming University of Science and Technology, \\ Kunming, China. \\ ${ }^{3}$ Hunan Academy of Agricultural Science, Changsha, China. \\ ${ }^{4}$ College of Agriculture and Life Sciences, Cornell University, Ithaca, NY, USA. \\ ${ }^{5}$ Faculty of Animal Science and Technology, Yunnan Agricultural University, Kunming, China. \\ ${ }^{6}$ Zhejiang Academy of Agricultural Science, Hangzhou, China.
}

\begin{abstract}
Cytoplasmic male sterility (CMS) is a maternally inherited trait that derives from the inability to produce functional pollen in higher plants. CMS results from recombination of the mitochondrial genome. However, understanding of the molecular mechanism of CMS in pepper is limited. In this study, comparative transcriptomic analyses were performed using a near-isogenic CMS line 14A (CMS-14A) and a maintainer line 14B (ML-14B) as experimental materials. A total of 17,349 differentially expressed genes were detected between CMS-14A and ML-14B at the PMC meiosis stage. Among them, six unigenes associated with CMS and 108 unigenes involved in energy metabolism were identified. The gene orf165 was found in CMS-14A. When orf165 was introduced into ML-14B, almost $30 \%$ of transgenic plants were CMS. In addition, orf165 expression in transgenic CMS plants resulted in abnormal function of some genes involved in energy metabolism. When orf165 in transgenic CMS plant was silenced, the resulted orf165-silenced plant was male fertile and the expression patterns of some genes associated with energy metabolism were similar to ML-14B. Thus, we confirmed that orf165 influenced CMS in pepper.
\end{abstract}

Keywords: Capsicum annuum L., comparative transcriptomic analyses, transgenic plants, mitochondrial targeting, VIGS.

Received: January 23, 2021; Accepted: July 04, 2021.

\section{Introduction}

Pepper (Capsicum annuum L.) is originally from Central and South America. It is one of the most important vegetables in the world (Zou, 2002). Pepper has very obvious heterosis. At present, commercial hybrid pepper seeds are mainly produced by manual emasculation and artificial pollination (Zou, 2002). Artificial production of hybrid seeds is not only laborious and time-consuming, which increases the cost of hybrid seeds, it also makes it difficult to guarantee the purity of seeds (Dhaliwal and Jindal, 2014). The use of cytoplasmic male sterility (CMS) and insect pollination reduces the labor required, and the cost of hybrid seeds by $50 \%$ (Zou, 2002). CMS also improves the purity of $F_{1}$ seeds because self-pollination does not occur. As a result, researchers and breeders are increasingly using CMS and studying its application in hybrid seed production (Dhaliwal and Jindal, 2014).

CMS is a maternal hereditary trait, and CMS plants cannot produce functional pollen grains. Mitochondria are energy-producing organelles, and CMS is generally thought to result from rearrangement of the mitochondrial genome

Send correspondence to Minghua Deng. College of Horticulture, Yunnan Agricultural University, Kunming 650224, China. E-mail: dengminghua2013@sina.com.

\#Those authors contributed equally to this work. due to the production of a novel open reading frame (ORF) (Hanson and Bentolila, 2004; Linke and Börner, 2005; Kim et al., 2007; Yang et al., 2010). The new mitochondrial ORF consists of fragments derived from other genes, or by deletion of normal mitochondrial genes, such as urf13 in maize CMS-T (Dewey et al., 1986) and orf220 in stem mustard (Yang et al., 2010). The relationship between CMS-related ORFs and CMS occurrence has been confirmed in some experiments (Hanson and Bentolila, 2004). In sunflower, transgenic plants expressing the CMS-related orfH522 gene were male sterile. In addition, transgenic sterile plants showed abnormalities in the tapetum cell layer. In the anther of sterile plants, premature DNA fragmentation and programmed cell death were observed during meiosis (Nizampatnam et al., 2009). In Brassica juncea, the mitochondrial targeting sequences of the CMS-associated $\operatorname{orf} 220$ and the $\beta$-subunit of the $F_{1}$ ATPase induced male sterility. In addition, abnormal flower development was observed in transgenic plants (Yang et al., 2010). In some cases, targeting novel ORFs expressed in mitochondria can lead to male sterility or semi-sterility (Duroc et al., 2006; Kim et al., 2007; Yamamoto et al., 2008; Wang et al., 2016). Most ORFs in the CMS line cause abnormalities energy metabolism in CMS. Molecular studies have shown that changes in the $\mathrm{F}_{1} \mathrm{~F}_{0}$-ATP synthase subunit, such as from Honglian rice ATP6 and sunflower ATP8 and ATPA, may induce CMS in plants (Sabar et al., 2003; Yang et 
al., 2009a,b; Zheng et al., 2012). For example, orf79 in rice encodes a cytotoxic peptide co-transcribed with the B-atp 6 gene (Wang et al., 2016), and orf522 in sunflower encodes a protein homologous to ATP8 in Reclinomonas (Balk and Leaver, 2001). Furthermore, male sterility can be induced by mutation of a gene that encodes energy-metabolizing enzyme subunits. For example, in the Arabidopsis $M G P 1$ gene, the $F_{\mathrm{A}} d$ subunit mutation of $F_{1} F_{0}$-ATP synthase causes pollen grains to die (Li et al., 2010).

In pepper, CMS was first seen in C. annuum accession PI164835 (Peterson, 1958). Studies have shown that the gene orf456 is related to CMS of pepper (Kim et al., 2007). Expression of orf456 in transgenic Arabidopsis resulted in male sterility in half of the T1 transgenic population (Kim et al., 2007). The pseudogene Yatp6-2 is related to Capsicum CMS (Kim and Kim, 2006). Male sterility was observed in the maintainer line in which atp6-2 was silenced (Ji et al., 2014); however, silencing $\Psi$ atp6-2 in the CMS line resulted in fertility recovery. The altered transcript orf507 is reportedly related to CMS in pepper (Gulyas et al., 2010). The orf507 encodes a toxic protein that interacts with a subunit of ATP synthase 6 to inhibit cell growth (Li et al., 2013). The expression of orf507 in the pepper maintainer line resulted male sterility in transgenic plants (Ji et al., 2015).

Using next-generation sequencing technology (RNASeq), whole transcriptome sequencing is a convenient way to study gene expression at the genome-wide level and to define presumed gene functions. In recent years, many studies have demonstrated the efficiency and sensitivity of RNA-Seq in variety of biological contexts (Liu et al., 2013; Martínez-López et al., 2014). Rapid progress has been made in understanding transcriptional procedures related to the specific developmental processes of many plant species, but little study has been conducted in pepper (Liu et al., 2013; Martínez-López et al., 2014). There is a great opportunity to do this kind of research using RNA-Seq in pepper.

CMS line 14A(CMS-14A) was generated by backcrossing several generations of 14B natural CMS mutants. Microscopic examination showed that the anthers in CMS-14A were abnormally developed during meiosis of pollen mother cells and did not produce fertile pollen. However, little is known about the molecular mechanisms of CMS in CMS-14A. We report here our investigation of the CMS-related gene orf165 in pepper 14A and demonstrate that when introduced with the mitochondrial targeting sequence it produced a male-sterile phenotype in transformed peppers; and silencing orf165 in transgenic male-sterile lines resulted in pollen fertility. Based on this evidence, we propose that orf165 is a potent candidate gene for determining the male sterility phenotype of CMS in pepper.

\section{Material and Methods}

\section{Plant material}

Near-isogenic C. annuum CMS-14A (S/rf/rf genotype) (A) and ML-14B (N/rf/rf) (B) were used in this study. These were planted at the Experimental Station of Yunnan Agricultural University in Kunming, China. Flower buds at the developmental stage of the spore cell division stage (stages A1 and B1) and pollen mother cell (PMC) meiosis stage (stages A2 and B2) were selected for RNA-Seq analysis.

\section{Cytological observation}

Cytological observation of flower bud development was performed by optical microscopy reported by Lee et al. (2008).

\section{RNA extraction and library preparation for transcriptome analysis}

Total RNA isolated from A1, B1, A2 and B2 was used to construct a sequence library. Prior to library construction, RNA quality and quantity were verified and treated with DNase I. After purification of the poly-(A) mRNA, adaptor-ligated fragments were generated, and the desired cDNA fragment range $(200 \pm 25 \mathrm{bp})$ was excised from the gel. The cDNA fragment was amplified and sequenced with Solexa using the Illumina HiSeq2000 sequencing platform enriched by the Beijing Genomics Institute (Shenzhen, China) (MartínezLópez et al., 2014).

\section{De novo assembly, assessment, and annotation}

After filtering the sequence data, transcriptome assembly was performed using Trinity short read assembly software. The unigene sequences were blastx-aligned with the protein databases NR, Swiss-Prot, KEGG, and COG (e-value < 0.00001) (Natale et al., 2000), and the best alignment of the proteins was used to determine the sequence orientation of unigenes. For unigenes that were not comparable within the above four libraries, we used ESTScan software to determine the sequence direction (Iseli et al., 1999; Conesa et al., 2005).

\section{Differential expression gene (DEG) analysis and validation}

The KEGG database and related software applications (http://www.genome.jp/kegg/kegg4.html) were used to analyze metabolic pathways (Nakao et al., 1999; Kanehisa et al., 2008). The transcript expression was calculated by the RPKM method using the following formula: $\operatorname{RPKM}(\mathrm{A})=10^{6} \times \mathrm{C} /\left(\mathrm{N} \times \mathrm{L} / 10^{3}\right)$ (Mortazavi et al., 2008). The DEGs and P-values between the two samples were screened by the method of Audic and Claverie (1997), and the threshold of the P-value in multiple tests was determined by FDR method. We used "FDR $\leq 0.001$ and the absolute value of $\log 2$ Ratio $\geq 1$ " as a threshold for judging the significance of gene expression differences.

Fourteen DEGs related to energy metabolism and one male sterility-related gene were selected for quantitative real time PCR (qRT-PCR) validation.

\section{Physiological-biochemical characteristic analysis}

The amount of ATP was determined using the method of John (1970) and Wang et al. (2007). The $\mathrm{H}^{+}$-ATPase activity was estimated according to Bisswanger (2005). BiFC assays were performed according to Waadt et al. (2008).

\section{Overexpression of orf165 in ML-14B}

To construct the vector, a mitochondrial transport sequence from yeast (encoding the first 25 amino acids of coxIV) was cloned (Kim et al., 2007). The coxIV pre-sequence and orf165 sequence were then cloned into the pRI101 vector 
to obtain the 35S-CXORF (pRI101:35S-coxIV-orf165) for plant transformation (Ji et al., 2015). The A. tumefaciensmediated transformation was performed according to Clough and Bent (1998) except for some minor changes as follows: when Agrobacterium tumefaciens of $\mathrm{OD}_{600}$ was $0.4-0.6$, the cells were collected by centrifugation; they were resuspended with $5.0 \%$ sucrose $+0.1 \%$ silwet $77+0.1 \mathrm{mmol} \cdot \mathrm{L}^{-1} \mathrm{AS}$ as a suspension to an $\mathrm{OD}_{600}$ value of 0.5-1.0. After the red fruits were ripe, the seeds were harvested. Antibiotic selection was applied during seed germination and PCR used to identify seedlings grown from resistant seeds. In transgenic pepper plants, 6-8 leaf stage seedlings were used for molecular detection of the $\operatorname{orf1} 165$ cassette.

\section{Southern blotting}

Southern blots were performed using the PCR DIG Probe Synthesis Kit and the DIG Luminescence Detection Kit according to the manufacturer's instructions.

\section{VIGS orf165 in transgenic male-sterile plants}

The pTRV vector and A. tumefaciens strain GV3101 were prepared for VIGS as described by Lacomme et al. (2003). Transgenic plants were grown by cutting propagation (Zhu et al., 2007). There were 50 cut seedlings and four true leaves used for VIGS inoculation assay. At the 6-8 leaf stage, seedlings were used for molecular detection of VIGS at the RNA level. Seedlings whose expression of orf165 was significantly lower than CK were identified as positive plants of VIGS; otherwise, they were identified as non-silent plants according to Ji et al. (2014).

\section{Morphological analysis of pollen grains}

Pollen abundance and pollen characteristics were scored after flowering. Anthers from 10 randomly selected flowers were collected, observed, and photographed under an optical microscope. Pollen abortion was tested by $\mathrm{I}_{2}-\mathrm{KI}$ staining (Wang et al., 2016).

\section{qRT-PCR analysis of orf165 and genes involved in energy metabolism}

Total RNA was isolated from flower buds in spore cell division (stage 1), PMC meiosis (stage 2), mononuclear microspores (stage 3 ) and mature pollen stage (stage 4). First strand cDNA was synthesized and qRT-PCR performed as described in our previous study (Deng et al., 2018). Relative gene expression was calculated using the $2^{-\Delta \Delta C t}$ method (Livak and Schmittgen, 2001). All primers used are listed in Table S1. All experiments were repeated three times and ACTIN was used as a reference gene.

\section{Results}

\section{Comprehensive analysis of pollen grains and energy metabolism between CMS-14A and ML-14B}

ML-14B produced many pollen grains (Figure 1A) but CMS-14A produced none (Figure 1B). $\mathrm{I}_{2}-\mathrm{KI}$ stained the ML-14B pollen grains and they appeared round and dark (i.e. viable) (Figure 1E).

At the meiosis stage of the PMC, the size of tapetal cells of ML-14B increased and they became highly vacuolated (Figure 2B). In CMS-14A, meiosis of the PMC mother cell occurred and the tetrads were formed in the locule, but the tapetal cells were abnormally elongated, bulged, and highly vacuolated; because the locule could not expand, the tetrads were squeezed together (Figure 2A).

The ATP content in the buds of CMS-14A and ML14B showed a continuous rising trend, but was significantly lower in CMS-14A than in ML-14B for all stages (Figure 3). The $\mathrm{H}^{+}$-ATPase activity was higher in CMS-14A than in ML-14B (Figure 3).
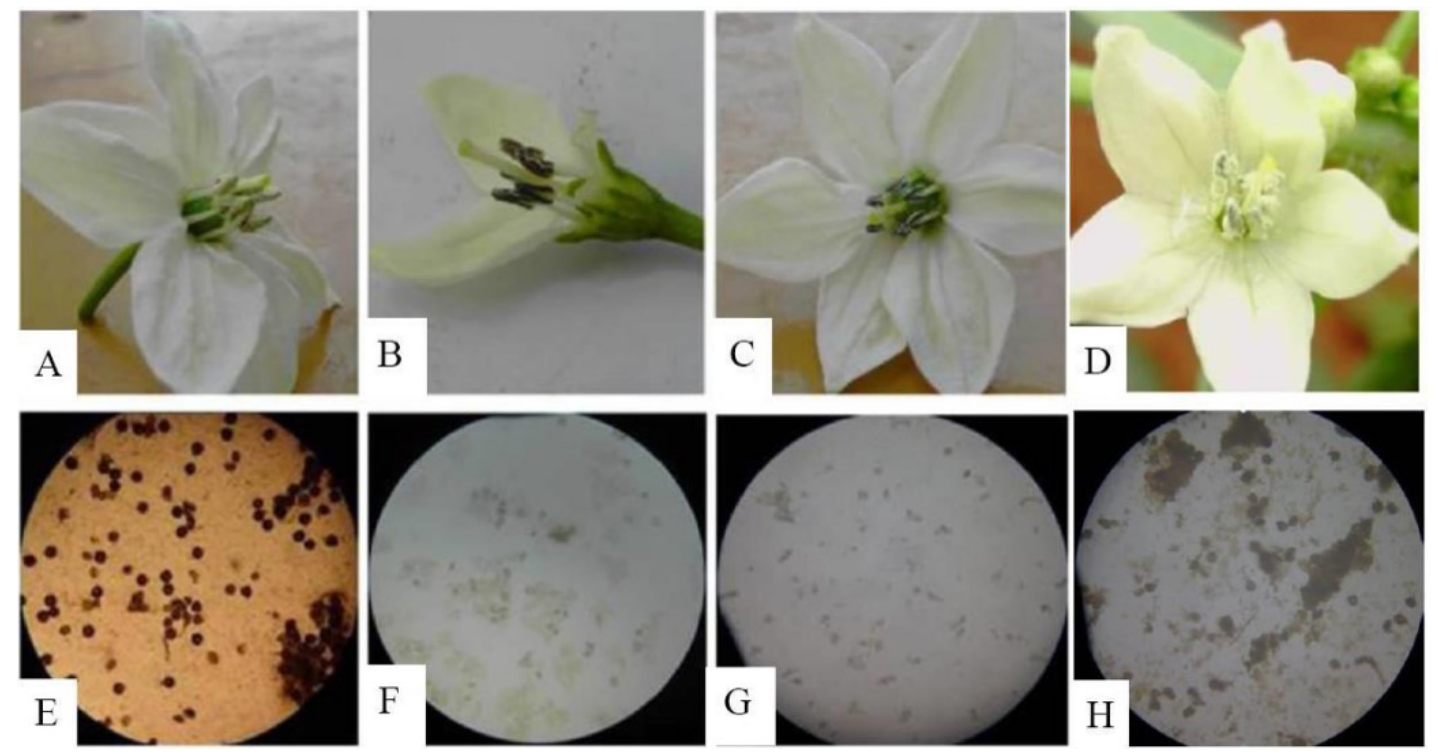

Figure 1 - Morphology and pollen viability of TP6, SP23, CMS-14A, and ML-14B. A: Blooming flowers of ML-14B had many pollen gains on the stamen; B: blooming flowers of TP6 had few pollen gains on the stamen; C: blooming flowers of CMS-14A had no pollen gain on the stamen; D: blooming flowers of SP23 had plenty of pollen gains on the stamen; E: most pollen gains from ML-14B appeared round and dark stained with $\mathrm{I}_{2}-\mathrm{KI}$; F: most pollen gains from TP6 appeared empty and deflated stained with $\mathrm{I}_{2}-\mathrm{KI}$; G: no pollen gain from CMS-14A was found stained with $\mathrm{I}_{2}-\mathrm{KI}$; H: most pollen gains from SP23 appeared round and dark stained with $\mathrm{I}_{2}-\mathrm{KI}$. 


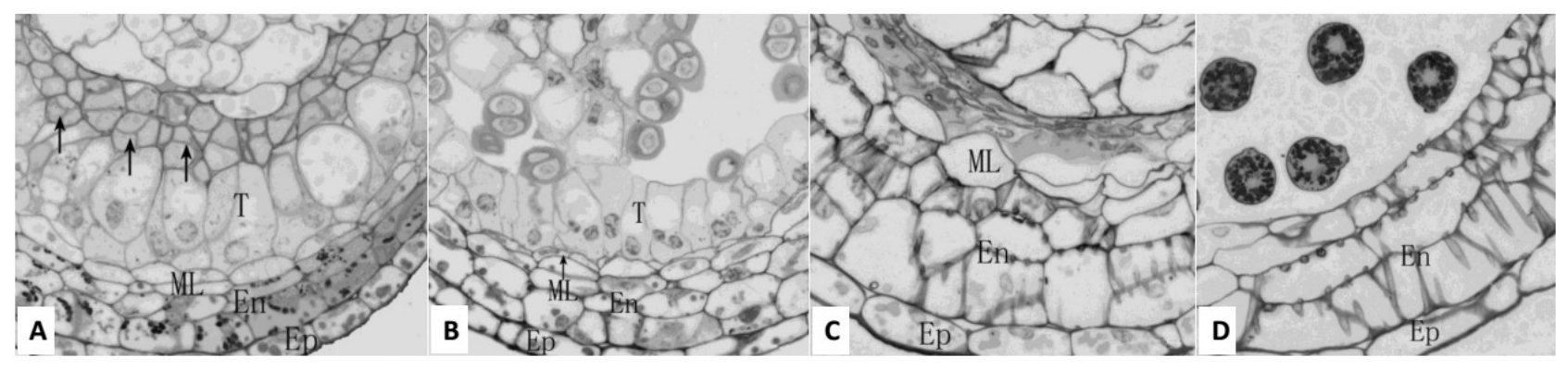

Figure 2 - Cytological observation of anthers of cytoplasmic male sterility line (CMS-14A), maintainer line (ML-14B), transgenic male-sterile plant (TP6), and transgenic male-fertility plant (SP-23) (200×). Ep: epidermic cells; En: endothecium cells; T: tapetal cells; ML: middle layer cells. (A). In sterile anthers of CMS-14A (sterility line) at the stage of tetrad microspore, locule did not enlarge and tetrad microspores were extruded and agglutinated (arrows). (B). The fertile anther of ML-14B (maintainer line) at the tetrad stage showed tetrad microspores in locule and anther wall, in which the size of T cells increased and became highly vacuolated. (C). At the time near anthesis, sterile anthers of TP6 (transgenic male-sterile plant) had four empty locules in which only some vestiges of aborted pollen and tapetal cells were apparent. The ML cells were still intact, the wall of En cells showed fiber thickening. (D). In the fertile anther of SP23 (transgenic male-fertility) just before anthesis, both T cells and ML cells degenerated completely and there was one layer of Ep cells and three layers of En cells in the anther wall.
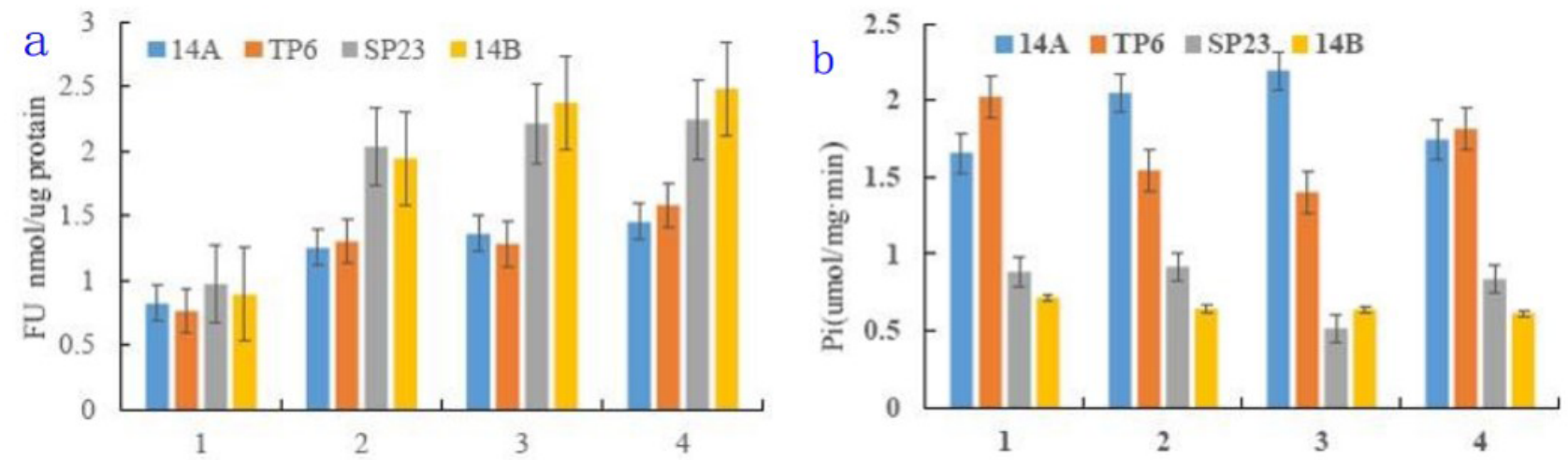

Figure 3 - ATP contents and $\mathrm{H}^{+}$-ATPase activities in TP6, SP23, CMS-14A, and ML-14B. 1: The spore cell division stage; 2: pollen mother cell meiosis stage; 3: mononuclear microspores; 4: mature pollen stage.

\section{Flower transcriptome sequence and analysis of DEGs}

Flower transcriptome sequencing output and de novo sequence assembly

More than $52 \times 10^{6} 49$-nt clean reads were generated from different development stage of pepper flowers of CMS14A and ML-14B. Total clean lengths exceeded $47 \times 10^{6}$, and the Q20 alkali content exceeded 97\% (base quality value $>$ 20) (Table S2).

Using Trinity, the high quality reads were de novo assembled into $140,561,152,560,147,640$, and 127,553 contigs from A1, A2, B1, and B2, respectively. A total of 97,475 single genes with a total length of 93,202,979 nt were obtained from all libraries.. The size distribution of the pepper flower contigs and unigenes was shown in Table S3.

Using the BLASTX program (E-value $<10^{-5}$ ), approximately 60,000 significant BLAST hits were obtained (Figure S1). The ESTScan can be used to predict if a unigene CDS does not have a BLAST hit (Iseli et al., 1999); more than 2000 unigenes were analyzed using this method (Figure S2).

\section{Functional annotation}

BLASTX was used to search distinct gene sequences against the $\mathrm{Nr}$ database, and found 61,365 unigenes (63.0\% of all 97,475 unigenes) with hits exceeding the E-value cutoff.
Similarly, 37,039 unigenes $(38.0 \%)$ were identified from the SwissProt database. A total of 76,833 unigenes were annotated in one or more of the databases $(78.8 \%)$, suggesting that they had relatively conserved functions.

Among 61,365 $\mathrm{Nr}$ hits, more than 40,000 sequences were classified by COG and distributed among $25 \mathrm{COG}$ categories (Figure S3). Of the 25 COG categories, "General function prediction only" was the largest category, followed by "Replication, recombination and repair," and "Transcription." The smallest category were "Nuclear structure" and "Extracellular structures."

Using BLAST2GO, we assigned more than $3.0 \times$ $10^{5}$ unigenes and grouped the terms into the three main GO categories and 55 sub-categories (functional groups) (Figure S4). In each of the three main categories (biological processes, cellular components, and molecular functions), the main terms were "Cellular process," "Metabolic process," and "Cell." About half of the genes fell into the "Biological processes" category.

We mapped the annotated sequences to the reference canonical pathways contained in the KEGG database. In all, nearly 35,000 unigenes were assigned to $128 \mathrm{KEGG}$ pathways. The most representative category was "Metabolic pathways", and the "Biosynthesis of secondary metabolites" and "Plantpathogen interaction" pathways were also well represented. 
Statistical analysis of DEGs in CMS-14A and ML-14B during flower development

Based on gene expression profiles, we identified the DEGs between CMS-14A and ML-14B using FDR $\leq 0.001$ and the absolute value of $\log _{2}$ ratio $\geq 1$ as the threshold. As a result, a large number of DEGs were obtained between the A1-vs.-B1, A2-vs.-A1, B2-vs.-B1, and A2-vs.-B2 (Figure 4I).

Because a CMS may be the result of the interactions between several genes with different biological functions, pathway analysis provides a better functional insight into DEGs and identifies the key genes involved. In B2-vs.-B1, A1-vs.-B1, A2-vs.-A1 and A2-vs.-B2, there were 8,955, $2,448,4,249$, and 17,349 DEGs identified, respectively, and these were assigned to $51 \mathrm{GO}$ categories (Figure S5). Among the genes associated with biological processes, $14.9 \%$ were associated with cellular processes, $14.3 \%$ with metabolic processes, $11.2 \%$ with single-organism processes, and 7.6\% with response to stimuli in A2-vs.-B2. Among the cellular component, genes encoding cell and cell part were dominant (24.6\%), followed by organelles (19.3\%) and membranes (10.2\%). Catalytic activity (44.1\%) and binding (42.2\%) were the main functional groups.

\section{Analysis of DEGs associated with CMS and energy metabolism in pepper flower and qRT-PCR validation}

There were 108 unigenes identified as involved in energy metabolism (Table S4). In A2-vs.-B2, B2-vs.-B1, A2-vs.-A1, and $\mathrm{A} 1$-vs.-B1, there were $30,57,48$, and 48 unigenes upregulated and $15,12,19$, and 24 down-regulated, respectively (Table S5, S6, S7, S8).

Fourteen unigenes involved in energy metabolism and one unigene associated with CMS were selected for qRT-PCR analysis. The 14 unigenes were putatively the ATP synthase beta subunit (ATP2), ATP synthase subunit delta (ATP4), ATP synthase subunit 6 (ATPO), ATP synthase subunit alpha (ATPA), cytochrome oxidase subunit 2 (coxII), cytochrome oxidase subunit III (coxIII), mitochondrial nad2, mitochondrial nad7, apocytochrome $b(\mathrm{cob})$, aconitate hydratase, succinate dehydrogenase, and pyruvate kinase. The expression patterns of the selected unigenes were basically consistent with Illumina sequencing (Figure 5).

\section{Characterization of ORF165 from CMS-14A}

Six unigenes associated with CMS were identified (Table S4). Compared with ML-14B, CL10138.Contig1, CL1641.Contig1, and CL1641.Contig4 in CMS-14A were down-regulated, but CL10138.Contig2 was up-regulated. Unigene22461 was not expressed in CMS-14A, but was highly expressed in ML-14B. Unigene18482, with known protein function of CMS, was uniquely expressed in CMS$14 \mathrm{~A}$, indicating that it may be related to CMS in pepper, so it was selected for further study.

BLAST search showed that the nucleotide and protein sequences of this fragment (Unigene18482) showed high similarity (99\% and 98\%) to orf507 (Accession No. FJ175153 and ACJ74044.1) in CMS pepper. According to the previous results of orf507, total DNA extracted from CMS-14A and ML-14B was used for PCR. The expected size (about 600 bp) of a single PCR product was obtained in CMS-14A, but no bands were detected in ML-14B. After cloning and sequencing the PCR fragment, a 498-bp ORF encoding 165 amino acids was identified and named orf165. Compared with orf 507 , nine nucleotide deletions were detected at the +22 to +30 bp position, and three point-mutations were detected at $+34,+39$, and +42 bp positions in orfl $65:+39$ and +42 bp were silent; and at $+34 \mathrm{bp}$, lysine (AAA) became glutamine (CAA) (Figure S6). It was predicted that the deletion of nine nucleotides would shorten the orf 507 product to 165 amino acids. Multiple alignments showed that orf165 and orf507 shared $99 \%$ nucleotide sequence identity.

The total base composition of orf $165 \mathrm{CDS}$ was A $31.12 \%$ (155), G 19.88\% (99), T 32.13\% (160), and C $16.87 \%$ (84).

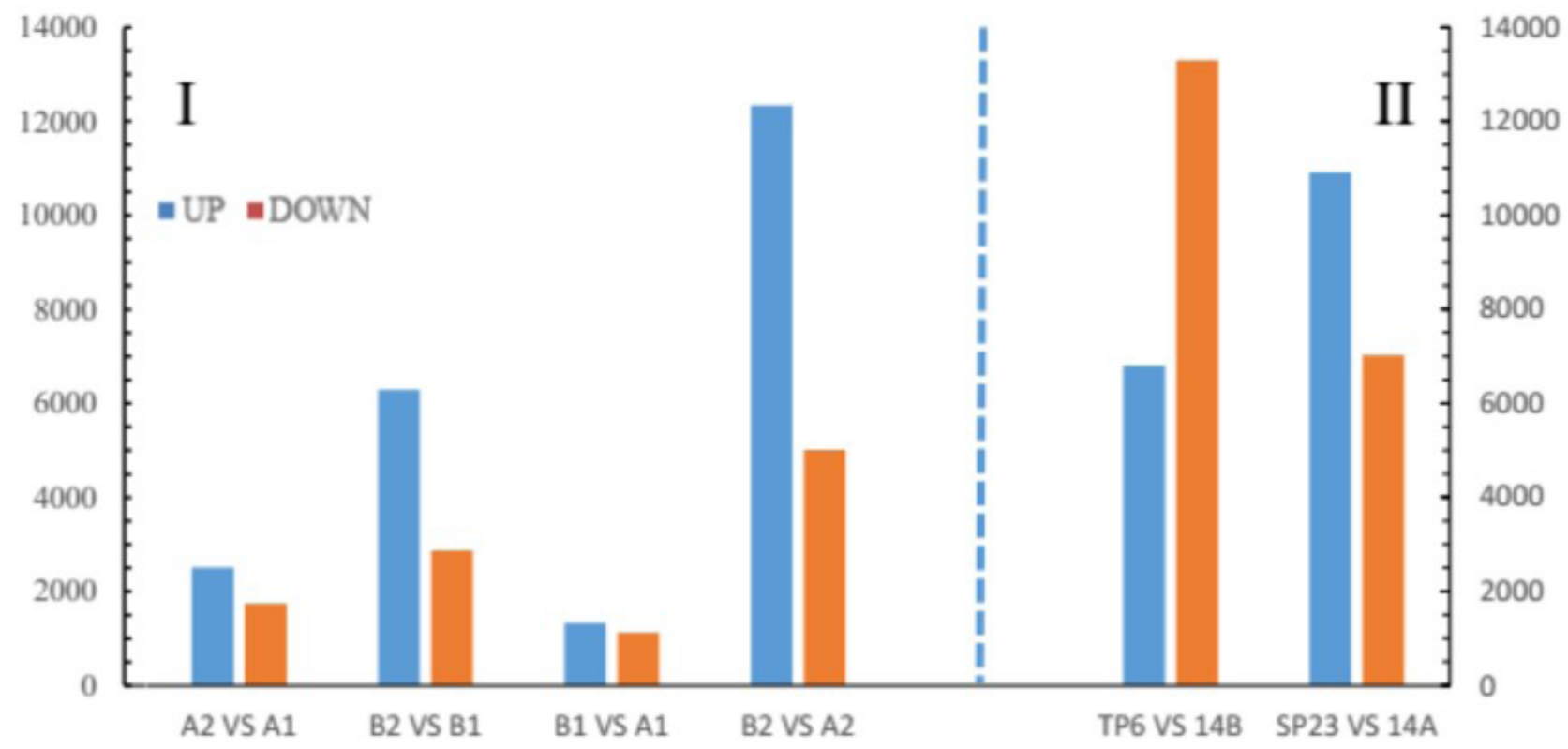

Figure 4 - Differentially expressed genes (DEGs). 

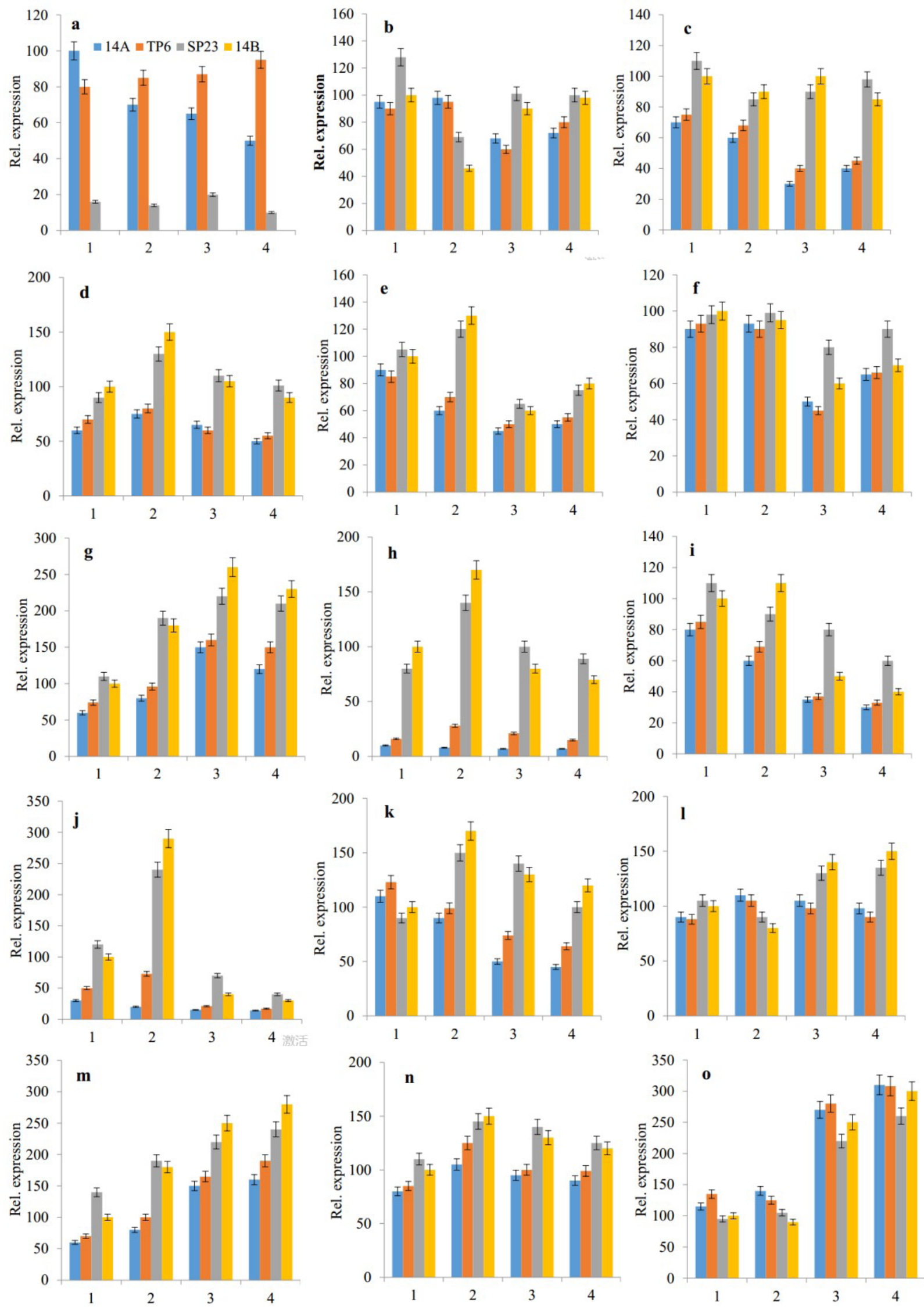

Figure 5 - The qRT-PCR analysis of selected CMS-related gene and energy metabolism related genes in CMS-14A, TP6, SP23, and ML-14B. 1: the spore cell division stage; 2: pollen mother cell meiosis stage; 3: mononuclear microspores; 4: mature pollen stage. A: Unigene18482 All; B: Unigene13850 All; C: Unigene11869_All; D: CL5535.Contig2_All; E: CL9996.Contig2_All; F: Unigene2101_All; G: CL420.Contig2_All; H: CL5464.Contig1_All; I: Unigene28657 All; J: CL11399.Contig2 All; K: CL11665.Contig4 All; L: Unigene24327 All; M: CL367.Contig2 All; N: Unigene33404 All; O: CL6833.Contig3_All. 
The theoretical $\mathrm{pI}$ and the molecular mass of orf165 were 5.147 and $1.9109 \mathrm{kDa}$, respectively. The total number of negatively charged residues $(\mathrm{Asp}+\mathrm{Glu})$ in orf165 was 19. Total number of positively charged residues (Arg + Lys) in orf165 was 15 . The protein contained 876 carbon, 1325 hydrogen, 215 nitrogen, 249 oxygen, and 8 sulfur, with formula $\mathrm{C}_{876} \mathrm{H}_{1325} \mathrm{~N}_{215} \mathrm{O}_{249} \mathrm{~S}_{8}$. The total number of atoms was 2673 , extinction coefficient was 9065 (units of $\mathrm{M}^{-1} \mathrm{~cm}^{-1}$, at $280 \mathrm{~nm}$ measured in water), estimated half-life was $30 \mathrm{~h}$ (mammalian reticulocytes, in vitro), N-terminal of the sequence considered was $\mathrm{M}$ (Met), the calculated instability index (II) was 38.75 , and aliphatic index was 82.18 . The orf165 protein sequence was submitted to SignalP, and had no N-terminal signal peptide and was a non-secretory protein. A transmembrane region was predicted in orf165 (37-59AA). The secondary structure predicted by SOPMA indicated that the deduced orf 165 contained 69 alpha helices $(41.67 \%), 51$ extended strands $(30.95 \%), 14$ beta turns $(8.93 \%)$, and 31 random coils $(18.45 \%)$.

The cDNA from the buds in various developmental stages of CMS-14A and ML-14B was used as templates for qRT-PCR analysis to check orf165 expression. The orf165 was strongly expressed in CMS-14A, but not in ML-14B (Figure 5). The orf165 was highly expressed in flowers, but not in seeds, placentas, pericarps, roots, stems, and leaves.

It was reported that orf507 specifically interacted with MtATP6 (Li et al., 2013). The protein sequence of orf165 shared high similarity (98\%) with orf507 and so orf165 may also interact with MtATP6. The PCR was performed using total cDNA extracted from CMS-14A and ML-14B based on the MtATP6 sequence reported by Li et al. (2013). A single PCR product was obtained in CMS-14A and ML-14B. After cloning and sequencing the PCR fragment, a 168-bp ORF encoding 55 amino acids was identified. When plants were transformed with Agrobacterium harboring MtATP6 and orf165 fused to split halves of the yellow fluorescent protein, yellow fluorescence signals were observed in leaf tissues through confocal microscopy, indicating physical interaction between MtATP6 and orf165 proteins (Figure 6).

\section{Overexpressed orf165 in pepper leads to pollen abortion}

To determine the correlation between orf165 and the CMS characters in pepper, two constructs for ML-14B were performed. In the first construct (coxIV-orf165), the orf165 sequence was fused to the yeast's nuclear cox $I V$ pre-sequence (54 codons) for mitochondrial targeting of the protein. The second construct (coxIV-nonorf165) did not contain orf165.

The first (coxIV-orf165) and second (coxIV-nonorf165) constructs driven by $35 \mathrm{~S}$ gene promotor were used to transform ML-14B. The floral phenotype of the second (without orf165) transformants was normal and similar to that of controls (ML-14B). However, the anthers of coxIV-orf 165 plants exhibited a male-sterile phenotype. The pistils of anthers of coxIV-orf165 transformants developed normally; however, the pistils produced no pollen grains at the dehiscent stage.

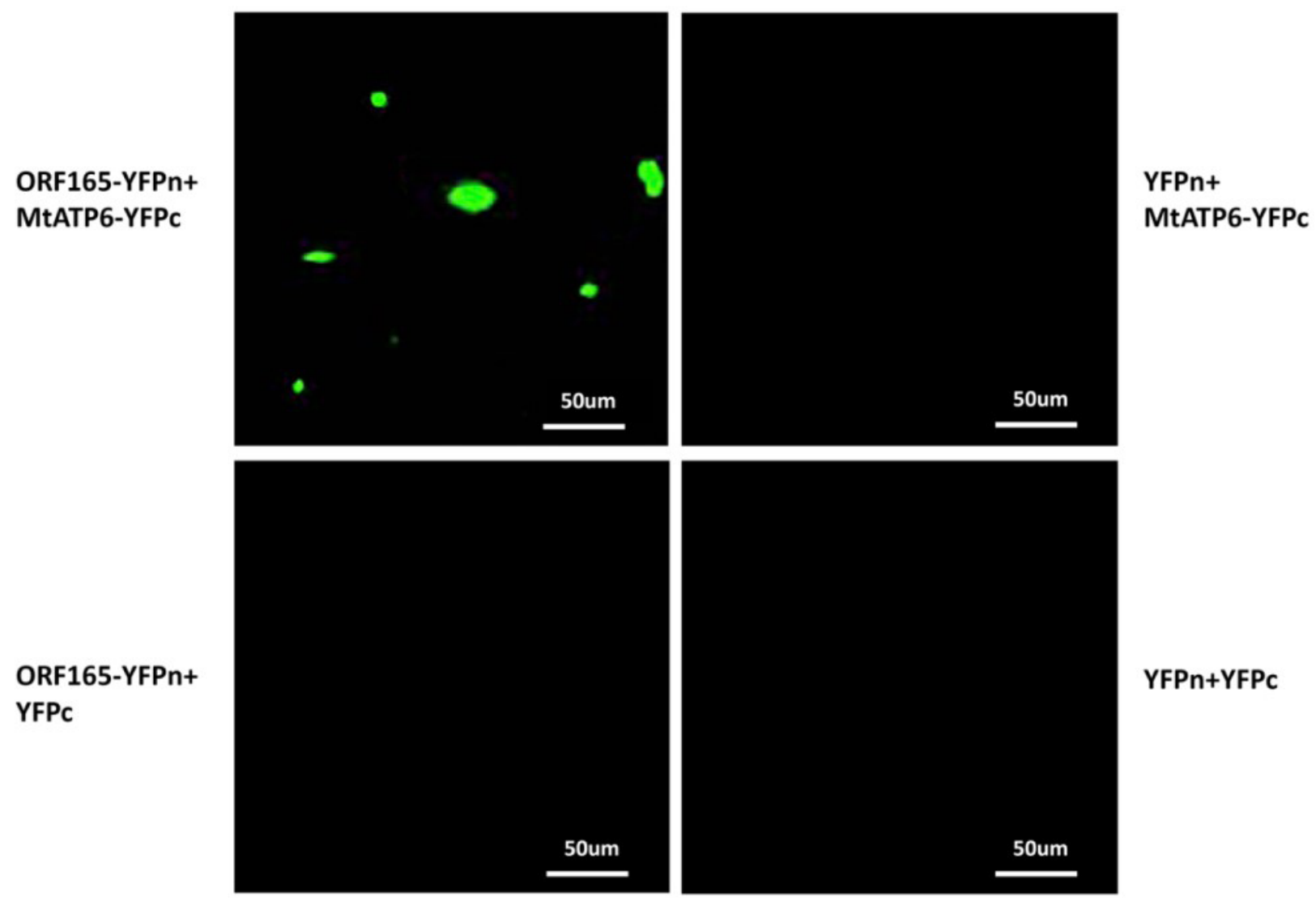

Figure 6 - Bimolecular fluorescence complementation (BiFC) assay for in vivo interaction of orf165 with MtATP6. YFP fluorescence was observed when ORF165:YFPn was co-expressed with MtATP6:YFPc. No YFP fluorescence was detected in control infiltration of ORF165:YFPn with YFPc, YFPn with MtATP6:YFPc, and YFPn with YFPc. 
About $30 \%$ of the total $\mathrm{T}_{1}$ transgenic plants (TPs) that contained coxIV-orf165 showed the male-sterile phenotype. Using the coxIV-orf165 plasmid as the positive control and the non-transgenic plant (ML-14B) as the negative control, PCR analysis with 17 kanamycin-resistant TPs was examined using gene-specific PCR primers. All TPs could amplify the objective fragment, but no PCR products were observed in non-transgenic plants (Figure S7A). The qRT-PCR analysis of these kanamycin-resistant TPs showed that orf165 was strongly expressed in male-sterile plants (TP1, 3, 4, 6, 8, 12,16 , and 17); TP2 was kanamycin resistant but slightly expressed the targeted orf165 and had a semi-male-sterile phenotype; several TPs (TP5, 7, 9, 10, 11, 13, 14, and 15) were kanamycin resistant but had a trace amount of targeted orf165 expression and a fertile phenotype indistinguishable from controls (Figure S7B).

TP6 was selected for analysis of pollen grains and gene expression. It produced few pollen grains (Figure 1C), while ML-14B produced many (Figure 1A) and CMS-14A produced none (Figure 1B). The pollen grains were stained with $\mathrm{I}_{2}-\mathrm{KI}$. Compared with the round and dark (i.e. viable) pollen grains of ML-14B, most pollen grains (77.7\%) from TP6 appeared empty and deflated (Figure 1E, G). This indicated that TP6 plant was semi-sterile, unlike CMS-14A with no pollen grains (Figure 1F).

The qRT-PCR analysis showed that orf165 expression increased slightly in TP6, decreased gradually in CMS-14A, and was absent from ML-14B throughout all stages of flower development (Figure 5). Southern blotting results showed three copies of orf165 in TP6 (Figure 7). The microscope image showed that large numbers of PMCs died inside the anthers of TP6 (Figure 2C).

\section{VIGS of orf165 restores pollen fertility}

We silenced orf165 in TP6 seedlings using VIGS and then analyzed the pollen abundance, pollen characteristics, and gene expression of VIGS plants.

The flower phenotype of TP6 plants inoculated with pTRV empty vector was abnormal and similar to TP6. However, the anthers of VIGS plants exhibited a male fertile phenotype (Figure 1D).

Fifty plants of TP6 inoculated with VIGS were selected for qRT-PCR assay. Of the 50 VIGS plants, $56 \%$ were identified as orf165-silenced positive plants (SP). The SP23 (no. 23 orf165 SP) slightly expressed the targeted orf165 and had a large number of pollen grains, with a male fertile phenotype indistinguishable from TP6.

SP23 was selected for analysis of pollen grains and gene expression. The results showed that SP23 had a large number of pollen grains (Figure 1D). Pollen grains of SP23 were stained with $\mathrm{I}_{2}$-KI (Figure $1 \mathrm{H}$ ). Most pollen grains of SP23 looked round and dark (i.e. viable), similar to those of ML-14B (Figure 1E).

Both tapetal cells (T) and middle layer cells (ML) degenerated completely and mature pollen grains formed in fertile anthers of SP23 just before anthesis (Figure 2D).

\section{TP6 14B PCR}

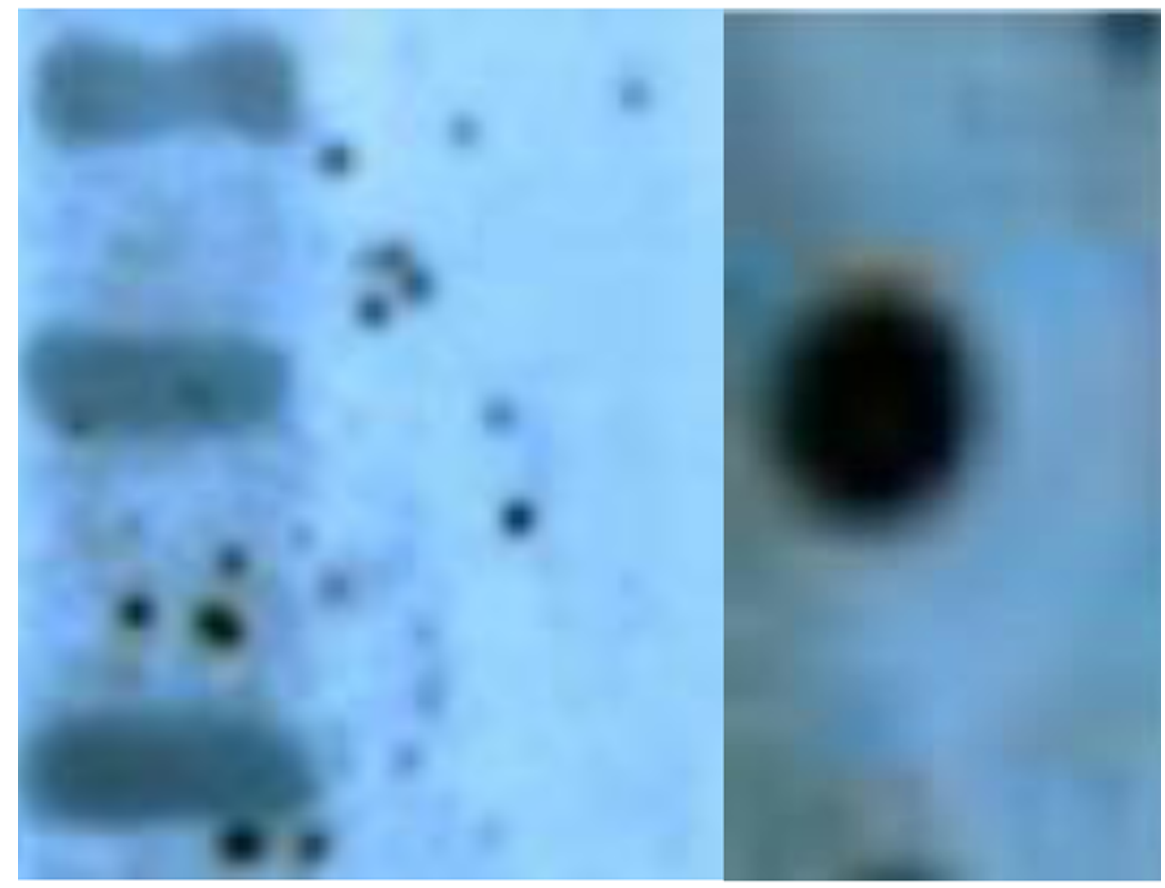

Figure 7 - Southern analysis of gDNA digested with EcoRI and HindIII hybridized with orfl65 probe to check copy number of exogenous gene in transgenic male-sterile plant (TP6). Of total gDNA, $10 \mu \mathrm{g}$ was digested with EcoRI and HindIII, separated in a $0.8 \%$ agarose gel, and transferred to a Hybond N+ membrane. The probe was digoxygenin (DIG-11-dUTP)-labeled 400-bp orf165. Southern blotting was performed using the PCR DIG Probe Synthesis Kit and DIG Luminescent Detection Kit according to the manufacturer's instructions. The orf165 showed three copies in TP6. ML-14B (a maintainer line of CMS 14A) was used as negative control and no band occurred in it; the PCR product of orf165 was used as a positive control. 


\section{Manipulating expression of orf165 affects energy metabolism of flower buds}

Using CMS-14A and ML-14B as controls, we analyzed ATP content and $\mathrm{H}^{+}$-ATPase activity in flowers of TP6 and SP23. The ATP contents in flowers of TP6 and CMS-14A showed similar trends and were significantly lower than those in ML-14B (Figure 3). The $\mathrm{H}^{+}$-ATPase activity in flowers of TP6 and CMS-14A was higher than that in ML-14B (Figure 3). The ATP levels in SP23 were similar to those in ML-14B, but much higher than in CMS-14A and TP6 (Figure 3). The $\mathrm{H}^{+}$-ATPase activity in flowers of SP23 was lower than in TP6 and CMS-14A and was similar to that in ML-14B (Figure 3).

The RNA-Seq analyses of TP6 and SP23 were integrated with transcriptomic profiling of CMS-14A and ML-14B at the PMC meiosis stage. The results showed that 6806 and 13,300 DEGs in the TP6 were up- or down-regulated, respectively, compared with the control (ML-14B). Compared with TP6, a total of 10,912 and 7,022 DEGs in SP23 were up- or downregulated, respectively. Compared with ML-14B, 5,009 and 12,340 DEGs in CMS-14A were up- or down-regulated, respectively. These results indicated that expression of orf 165 had a significant impact on global gene expression. The GO enrichment analysis classified three main categories: "Biological process", "Molecular function," and "Cellular component". In the "Molecular function" category, most DEGs were related to the "Catalytic activity" subcategory, while other DEGs were related to "Electron carrier activity" and "Enzyme regulator activity". The analyses indicated that orf165 might be related to energy metabolism.

We analyzed the expression of these genes in TP6 flowers using CMS-14A and ML-14B as controls (Figure 5). In TP6, throughout all stages of flower development, expression of CL6833.Contig3 (pyruvate kinase) was up-regulated gradually; while expressions of Unigene11869 (ATP4), CL5535. Contig2 (ATPO), CL9996.Contig2 (ATPA), Unigene2101 (COXII), CL420.Contig2 (COXIII), CL5464.Contig1 (NAD2), Unigene28657 (NAD7), CL11399.Contig2 (COB), CL11665.Contig4 (aconitase), Unigene24327 (succinate dehydrogenase), CL367.Contig2 (succinate dehydrogenase), and Unigene33404 (pyruvate kinase) decreased. Expression of Unigene13850 (ATP2) was up-regulated in the second stage and down-regulated in the first, third, and fourth stages. The expressions levels of 14 genes in TP6 were more similar to those in CMS-14A than those of ML-14B. The altered function of these 14 genes might be a factor that induced CMS in pepper.

The qRT-PCR was used to determine the expression of the 14 selected genes involved in energy metabolism in SP23 flowers (Figure 5). Unigene11869, CL5535.Contig2, CL420. Contig2, CL5464.Contig1, and CL11399.Contig2 expression in TP6 was down-regulated, but increased in SP23 and reached similar expression levels in ML-14B. Compared to TP6, the expression of CL6833.Contig3 (pyruvate kinase) in SP23 was down-regulated gradually. The expression levels of 14 genes in SP23 were more similar to those of ML-14B than TP6. These results indicated that orf165 expression was one factor causing pollen abortion in TP6 and CMS-14A.

\section{Discussion}

Near-isogenic lines combined with transcriptome sequencing is an effective method to screen for male sterility related genes

Near-isogenic lines combined with transcriptome sequencing can maximally eliminate genetic background variations, reduce false positives, and identify genes that control specificity more accurately and effectively (Liu et al., 2013; Chen et al., 2014). Near-isogenic lines have been widely used in the analysis of CMS in various plants in combination with transcriptome sequencing, and some CMSrelated genes have been found (Zheng et al., 2012; Liu et al., 2013; Chen et al., 2014; Yang et al., 2014; Wang et al., 2016). The material CMS-14A used in this study was produced by backcrossing several generations of natural CMS mutants of ML-14B (Zou, 2002). In this case, CMS-14A and ML-14B were a pair of typical near-isogenic lines with the exception of fertility. Transcriptome analysis of the buds indicated that the obtained DEGs were most likely related to CMS. In this study, we obtained a number of DEGs, including a total of 17,349 at the beginning of abortion (A2 vs B2 comparison).

We identified a total of six unigenes associated with CMS by functional annotation of DEGs. Among these genes, unigene 18482_All was shown to encode CMS-related protein by functional annotation, and was only expressed in CMS-14A. Further studies showed that unigene 18482_All only existed in CMS-14A, indicating that it was a new gene. Many studies have shown that amplification, rearrangement, and recombination of mitochondrial genes in higher plants leads to formation of new ORFs and CMS. The first CMS-related gene identified in higher plants was urfl3 in the mitochondrial genome of maize; specific genes associated with CMS were subsequently identified in the mitochondrial genomes of rice, sunflower, Brassica, radish, and pepper (Dewey et al., 1986; Singh and Brown, 1993; Dieterich et al., 2003; Kim and Kim, 2006; Wang et al., 2006; Kim et al., 2007; Ashutosh et al., 2008; Lee et al., 2009; Nizampatnam et al., 2009; Das et al., 2010; Jing et al., 2011).

Sequence alignment revealed that the nucleotide sequence of Unigene18482_All was highly similar (99\%) to the CMSrelated gene $\operatorname{orf} 507$ in mitochondria of CMS-417A in GenBank (Kim et al., 2007; Gulyas et al., 2010). Unigene18482_All had a nine-base deletion $(+22$ to +30$)$ and a three-base mutation in Unigene18482 All $(+34,+39$, and +42$)$. It is presumed to encode a 165-amino-acid peptide chain, and was named orf165. Male sterility lines from different origins had similar sequences, which have also been reported in Brassica; the genes $\operatorname{orf} 288$, orf286, and orf263 associated with male sterility in Brassica had high homology (Jing et al., 2011).

\section{Overexpression of orf165 induced male sterility of TPS}

To assess the relationship between orf165 and CMS, orf165 was introduced into ML-14B. Because orf165 is thought to be a mitochondrial gene, the pre-sequence of coxIV 
in yeast was used as a signal peptide for orf165 (Kim et al., 2007; Nizampatnam et al., 2009). The results showed that TPs with the coxIV pre-sequence transferred only showed normal fertility, but about $30 \%$ of TPs with coxIV-orf 165 transferred showed CMS. The anthers of TP6, a typical transgenic male-sterile plant, appeared dry and empty and did not normally break open to distribute pollen grains. The microscopic view of $\mathrm{I}_{2}-\mathrm{KI}$-stained anthers showed that most TP6 pollen grains were empty, indicating that orf165 caused CMS in transgenic pepper TP6. Introduction of orf 220 of Brassica into plants could result in CMS (Yang et al., 2010); and the introduction of orf507, a CMS-related gene of pepper lines, into Arabidopsis resulted in about $45 \%$ of transgenic Arabidopsis plants showing CMS (Kim et al., 2007). Our results are basically consistent with previous reports.

\section{Silencing of orf165 restores fertility of transgenic male plants}

To verify that CMS of TP6 was caused by orf165 expression, we silenced orf165 in TP6 by VIGS technology. SP23, a typical silenced plant, produced a large number of pollen grains, and $\mathrm{I}_{2}-\mathrm{KI}$ staining showed that pollen produced by SP23 was round and black, which was highly similar to ML-14B, indicating that orf165 silencing could lead to restoration fertility. Previously, according to reports, plants with silencing of the CMS-related gene $\Psi$ atp6-2 could form a lot of pollen, but the control plants did not form pollen (Ji et al., 2015). Our results are in line with previous reports.

\section{Expression of male sterility genes leads to abnormal energy metabolism in anthers}

The mitochondrial CMS-related genes interact with certain energy metabolism genes to reduce or inactivate enzyme activity and disrupt energy supply balance, resulting in CMS (Sabar et al., 2003). The CMS-related gene orf507 of pepper caused microspore abortion by reducing the activity of ATP synthase (Li et al., 2013); Iatp6-2 can lead to a decrease in ATP synthase, which leads to CMS in pepper (Ji et al., 2014); in rice, WA352 inhibits expression of coxII, and orfH79, both of which resulted in abnormal mitochondrial $\mathrm{F}_{1} \mathrm{~F}_{0}$-ATPase activity, leading to CMS (Zhang et al., 2007; Luo et al., 2013). Mutation of MGP1 in Arabidopsis can increase the activity of ATP hydrolase, resulting in mitochondrial destruction and CMS (Li et al., 2010).

Through transcriptome analysis, we obtained a large number of DEGs in CMS-14A and ML-14B, which contained 108 encoding genes related to energy metabolism in mitochondria. Among them, 14 genes were verified by qRT-PCR and most of them were down-regulated in CMS14A. In orf165-overexpressing transgenic TP6, the expression patterns of these 14 genes were similar to that of CMS-14A, but significantly differed from its transgene receptor (ML14B). However, in SP23 plants whose orf165 was silenced, the expression patterns of these 14 genes were similar to that of ML-14B, but differed from its receptor (TP6). This may be because both CMS-14A and TP6 both contained a normally expressed orf165. The orf165 may interact with energy metabolism genes, resulting in expression imbalances; whereas in SP23, due to the abnormal expression of orf165, expression of the above 14 genes tended to be normal, and were consistent with ML-14B. Previous studies have shown that a pepper CMS-related gene, orf507, encodes a toxic protein that interacts with ATP6 and decreases ATP synthase activity, thereby causing CMS (Ji et al., 2014). In this paper, orf165 was highly homologous to orf507. The BiFC results showed that orf165 interacted with MtATP6. The $\mathrm{H}^{+}$-ATPase activity in CMS-14A and TP6 was higher than that in ML-14B and SP23. This suggested that the orf165 expression increased ATP hydrolysis activity, leading to energy supply imbalance and microspore abortion.

\section{Acknowledgements}

This study was supported by the Natural Science Foundation of China (Grant No 31760575) and the New Products Program of Yunnan Province (2018BB020).

\section{Conflict of Interest}

The authors declare that there is no conflict of interest that could be perceived as prejudicial to the impartiality of the reported research.

\section{Author Contributions}

This study was conceived by W-JF and Z-XX. The plant material preparation was carried out by Z-ZQ, Z-S, W-J, Y-TY, and Z-JR. D-MH, Z-K, S-GF, and H-JL performed the qRT-PCR. D-MH, L-JH, M-YQ, W-JL, and Z-HS analyzed the RNA-Seq data. D-MH, W-HJ, W-ZR, and W-JF performed the transgenic experiments and drafted the manuscript. Z-XX revised the manuscript. All authors read and approved the final manuscript.

\section{References}

Ashutosh PK, Kumar VD, Sharma PC, Prakash S and Bhat SR (2008) A novel orf108 co-transcribed with the atpA gene is associated with cytoplasmic male sterility in Brassica juncea carrying Moricandia arvensis cytoplasm. Plant Cell Physiol 49:284-289.

Audic S and Claverie JM (1997) The significance of digital gene expression profiles. Genome Res 7:986-995.

Balk J and Leaver CJ (2001) The PET1-CMS mitochondrial mutation in sunflower is associated with premature programmed cell death and cytochrome c release. Plant Cell 13:1803-1818.

Bisswanger H (2005) Practical enzymology. Chemical Industry Press, Beijing, China.

Chen P, Ran S, Li R, Huang Z, Qian J, Yu M and Zhou R (2014) Transcriptome de novo assembly and differentially expressed genes related to cytoplasmic male sterility in kenaf (Hibiscus cannabinus L.). Mol Breeding 34:1879-1891.

Clough SJ and Bent AF (1998) Floral dip: A simplified method for Agrobacterium- mediated transformation of Arabidopsis thaliana. Plant J 16:735-743.

Conesa A, Götz S, García-Gómez JM, Terol J, Talón M and Robles M (2005) Blast2GO: A universal tool for annotation, visualization and analysis in functional genomics research. Bioinformatics 21:3674-3676.

Das S, Sen S, Chakraborty A, Chakraborti P, Maiti MK, Basu A, Basu D and Sen SK (2010) An unedited $1.1 \mathrm{~kb}$ mitochondrial orfB gene transcript in the wild abortive cytoplasmic male sterility (WA-CMS) system of Oryza sativa L. subsp. indica. BMC Plant Biol 10:39. 
Deng MH, Wen JF, Zhao K, Huo JL, Zhang ZQ, Zhu HS and Zou XX (2018) A comprehensive view of expression profiles dynamics of capsaicinoid biosynthesis-related genes during pepper fruit development and under MeJA treatment. Pak J Bot 50:1013-1022.

Dewey RE, Levings $3^{\text {rd }} \mathrm{CS}$ and Timothy DH (1986) Novel recombinations in the maize mitochondrial genome produce a unique transcriptional unit in the Texas male-sterile cytoplasm. Cell 44:439-449.

Dhaliwal MS and Jindal SK (2014) Induction and exploitation of nuclear and cytoplasmic male sterility in pepper (Capsicum spp.): A review. J Hortic Sci Biot 89:471-479.

Dieterich J-H, Braun H-P and Schmitz UK (2003) Alloplasmic male sterility in Brassica napus (CMS 'Tournefortii-Stiewe') is associated with a special gene arrangement around a novel atp9 gene. Mol Genet Genomics 269:723-731.

Duroc Y, Gaillard C, Hiard S, Tinchant C, Richard B, Pelletier G and Budar F (2006) Nuclear expression of a cytoplasmic male sterility gene modifies mitochondrial morphology in yeast and plant cell. Plant Sci 170:755-767.

Gulyas G, Shin Y, Kim H, Lee J-S and Hirata Y (2010) Altered transcript reveals an orf507 sterility-related gene in chili pepper (Capsicum annuum L.). Plant Mol Biol Rep 28:605-612.

Hanson MR and Bentolila S (2004) Interaction of mitochondrial and nuclear genes that affect male gametophyte development. Plant Cell 16:S154-S169.

Iseli C, Jongeneel CV and Bucher P (1999) ESTScan: A program for detecting, evaluating, and reconstructing potential coding regions in EST sequences. Proc Int Conf Intell Syst Mol Biol 17:138-148.

Ji J-J, Huang W, Li Z, Chai W-G, Yin Y-X, Li D-W and Gong Z-H (2015) Tapetum-specific expression of a cytoplasmic orf507 gene causes semi-male sterility in transgenic peppers. Front Plant Sci 6:272.

Ji J-J, Huang W, Li D-W, Yin Y-X, Chai W-G and Gong Z-H (2014) ACMS-related gene, $\psi$ atp6-2, causes increased ATP hydrolysis activity of the mitochondrial F1F0-ATP synthase and induces male sterility in pepper (Capsicum annuum L.). Plant Mol Biol Rep 32:888-899.

Jing B, Heng S, Tong D, Wan Z, Fu T, Tu J, Ma C, Yi B, Wen J and Shen J (2011) A male sterility-associated cytotoxic protein ORF288 in Brassica juncea causes aborted pollen development. J Exp Bot 63:1285-1295.

John JB St (1970) Determination of ATP in Chlorella with the luciferin-luciferase enzyme system. Anal Biochem 37:409-416.

Kanehisa M, Araki M, Goto S, Hattori M, Hirakawa M, Itoh M, Katayama T, Kawashima S, Okuda S, Tokimatsu T et al. (2008) KEGG for linking genomes to life and the environment. Nucleic Acids Res 36:D480-D484.

Kim DH and Kim B-D (2006) The organization of mitochondrial atp6 gene region in male fertile and CMS lines of pepper (Capsicum annuum L.). Curr Genet 49:59-67.

Kim DH, Kang JG and Kim B-D (2007) Isolation and characterization of the cytoplasmic male sterility-associated orf456 gene of chili pepper (Capsicum annuum L.). Plant Mol Biol 63:519-532.

Lacomme C, Hrubikova K and Hein I (2003) Enhancement of virus-induced gene silencing through viral-based production of inverted-repeats. Plant J 34:543-553.

Lee Y-P, Park S, Lim C, Kim H, Lim H, Ahn Y, Sung S-K, Yoon M-K and Kim S (2008) Discovery of a novel cytoplasmic male-sterility and its restorer lines in radish (Raphanus sativus L.). Theor Appl Genet 117:905-913.

Lee Y-P, Kim S, Lim H, Ahn Y and Sung S-K (2009) Identification of mitochondrial genome rearrangements unique to novel cytoplasmic male sterility in radish (Raphanus sativus L.). Theor Appl Genet 118:719-728.
Li J, Pandeya D, Jo YD, Liu WY and Kang B-C (2013) Reduced activity of ATP synthase in mitochondria causes cytoplasmic male sterility in chili pepper. Planta 237:1097-1109.

Li W-Q, Zhang X-Q, Xia C, Deng Y and Ye D (2010) Male gametophyte defective 1, encoding the FAd subunit of mitochondrial F1F0ATP synthase, is essential for pollen formation in Arabidopsis thaliana. Plant Cell Physiol 51:923-935.

Linke B and Börner T (2005) Mitochondrial effects on flower and pollen development. Mitochondrion 5:389-402.

Liu C, Ma N, Wang P-Y, Fu N and Shen H-L (2013) Transcriptome sequencing and de novo analysis of a cytoplasmic male sterile line and its near-isogenic restorer line in chili pepper (Capsicum annuum L.). PLoS One 8:e65209.

Livak KJ and Schmittgen TD (2001) Analysis of relative gene expression data using real-time quantitative PCR and the 2(-Delta Delta C (T)) method. Methods 25:402-408.

Luo D, Xu H, Liu Z, Guo J, Li H, Chen L, Fang C, Zhang Q, Bai M, Yao N et al. (2013) A detrimental mitochondrial-nuclear interaction causes cytoplasmic male sterility in rice. Nat Genet 45:573-577.

Martínez-López LA, Ochoa-Alejo N and Martínez O (2014) Dynamics of the chili pepper transcriptome during fruit development. BMC Genomics 21:143.

Mortazavi A, Williams BA, McCue K, Schaeffer L and Wold B (2008) Mapping and quantifying mammalian transcriptomes by RNA-Seq. Nat Methods 5:621-628.

Nakao M, Bono H, Kawashima S, Kamiya T, Sato K, Goto S and Kanehisa M (1999) Genome-scale gene expression analysis and pathway reconstruction in KEGG. Genome Inform Ser Workshop Genome Inform 10:94-103.

Natale DA, Shankavaram UT, Galperin MY, Wolf YI, Aravind L and Koonin EV (2000) Towards understanding the first genome sequence of a crenarchaeon by genome annotation using clusters of orthologous groups of proteins (COGs). Genome Biol 1:1-19.

Nizampatnam NR, Doodhi H, Narasimhan YK, Mulpuri S and Viswanathaswamy DK (2009) Expression of sunflower cytoplasmic male sterility-associated open reading frame, orfH522 induces male sterility in transgenic tobacco plants. Planta 229:987-1001.

Peterson PA (1958) Cytoplasmically inherited male sterility in capsicum. Am Nat 92:111-119.

Sabar M, Gagliardi D, Balk J and Leaver CJ (2003) ORFB is a subunit of F1F0-ATP synthase: Insight into the basis of cytoplasmic male sterility in sunflower. EMBO Rep 4:381-386.

Singh M and Brown GG (1993) Characterization of expression of a mitochondrial gene region associated with the Brassica 'Polima' CMS: Developmental influences. Curr Genet 24:316-322.

Waadt R, Schmidt LK, Lohse M, Hashimoto K, Bock R and Kudla J (2008) Multicolor bimolecular fluorescence complementation reveals simultaneous formation of alternative CBL/CIPK complexes in planta. Plant J 56:505-516.

Wang S, Wang C, Zhang X-X, Chen X, Liu J-J, Jia X-F and Jia S-Q (2016) Transcriptome de novo assembly and analysis of differentially expressed genes related to cytoplasmic male sterility in cabbage. Plant Physiol Biochem 105:224-232.

Wang Y, Addess KJ, Chen J, Geer LY, He J, He S, Lu S, Madej T, Marchler-Bauer A, Thiessen PA et al. (2007) MMDB: Annotating protein sequences with Entrez's 3D-structure database. Nucleic Acids Res 35:D298-D300.

Wang Z, Zou Y, Li X, Zhang Q, Chen L, Wu H, Su D, Chen Y, Guo J, Luo D et al. (2006) Cytoplasmic male sterility of rice with Boro II cytoplasm is caused by a cytotoxic peptide and is restored by two related PPR motif genes via distinct modes of mRNA silencing. Plant Cell 18:676-687. 
Yamamoto MP, Shinada H, Onodera Y, Komaki C, Mikami T and Kubo T (2008) A male sterility-associated mitochondrial protein in wild beets causes pollen disruption in transgenic plants. Plant J 54:1027-1036.

Yang J-H, Huai Y and Zhang M-F (2009a) Mitochondrial atpA gene is altered in a new orf220-type cytoplasmic male-sterile line of stem mustard (Brassica juncea). Mol Biol Rep 36:273-280.

Yang J, Liu X, Yang X and Zhang M (2010) Mitochondrially targeted expression of a cytoplasmic male sterility-associated orf220 gene causes male sterility in Brassica juncea. BMC Plant Biol 10:231.

Yang J-H, Zhang M-F and Yu J-Q (2009b) Mitochondrial nad2 gene is co-transcripted with CMS-associated orfB gene in cytoplasmic male-sterile stem mustard (Brassica juncea). Mol Biol Rep 36:345-351.

Yang P, Han J and Huang J (2014) Transcriptome sequencing and de novo analysis of cytoplasmic male sterility and maintenance in JA-CMS cotton. PLoS One 9:e112320.

Zhang H, Li S, Li P, Wan C, Chen Z and Zhu Y (2007) A honglian CMS line of rice displays aberrant F0 of F0F1-ATPase. Plant Cell Rep 26:1065-1071.

Zheng B-B, Wu X-M, Ge X-X, Deng X-X, Grosser JW and Guo W-W (2012). Comparative transcript profiling of a male sterile Cybrid Pummelo and its fertile type revealed altered gene expression related to flower development. PLoS One 7:e43758.

Zhu S, Cui Q and Liu W (2007) Research of in vitro cutting for fast-propagation on stem of hot pepper. Chinese Agri Sci Bull 23:274-277.

Zou XX (2002) China capsicum. China Agricultural Press, Beijing.

\section{Supplementary material}

The following online material is available for this article:

Figure S1 - Size distribution of CDS produced by searching unigene sequences against various protein databases.
Figure S2 - Size distribution of EST obtained from the ESTScan results.

Figure S3 - COG function classification of unigenes.

Figure S4 - GO function classification of unigenes.

Figure S5 - Histogram of GO classifications for differentially expressed genes.

Figure S6 - Multiple alignment of DNA sequences of orf165 and orf507.

Figure S7 - PCR and expression analysis of orf165 expression in 17 transgenic plants.

Table S1 - Oligonucleotides used in this study.

Table S2 - Output statistics for pepper flowers

Table S3 - Statistics of assembly quality for pepper flowers Table S4 - DEGs involved in energy metabolism and male sterility.

Table S5 - DEGs involved in energy metabolism in A2 vs B2 comparison.

Table S6 - DEGs involved in energy metabolism in B1 vs B2 comparison.

Table S7 - DEGs involved in energy metabolism in A1 vs A2 comparison.

Table S8 - DEGs involved in energy metabolism in A1 vs B1 comparison.

Associate Editor: Marcio C. Silva-Filho

License information: This is an open-access article distributed under the terms of the Creative Commons Attribution License (type CC-BY), which permits unrestricted use, distribution and reproduction in any medium, provided the original article is properly cited. 